\title{
Homogénéisation et H-mesures
}

\author{
Luc TARTAR
}

\section{Résumé.}

Ce texte décrit l'apparition des idées concernant l'Homogénéisation et les H-mesures, et suggère quelques aspects qui pourraient être utiles en Analyse Numérique.

\section{Mots clés.}

Homogénéisation, H-mesures.

\begin{abstract}
.
The text describes the apparition of ideas concerning Homogenization and $H$-measures, and suggests aspects which could be useful in Numerical Analysis.
\end{abstract}

\section{Keywords.}

Homogenization, $\mathrm{H}$-measures.

AMS Subject Classification. 35 B 27, 73 B 27

\section{Souvenirs}

Parce que je voulais être ingénieur, sans avoir aucune idée de ce que pouvait être le travail d'un ingénieur, j'avais préféré l'Ecole Polytechnique (encore sur la Montagne Sainte Geneviève à cette époque) à l'Ecole Normale Supérieure. J'eus ainsi la chance d'avoir deux enseignants remarquables, Laurent ScHWARTz comme professeur d'Analyse la première année, et Jacques-Louis Lions comme professeur d'Analyse Numérique la seconde année. Un soir de l'automne 1966, au début de ma deuxième année d'études, j'allais écouter une conférence que Laurent SCHWARTz donnait pour les élèves dans l'amphi PoINCARÉ où il avait enseigné l'année précédente (les cours de Jacques-Louis Lions avaient lieu dans l'amphi GAY-LussaC). Sa conférence avait un titre comme "le devoir et la responsabilité du scientifique", mais il y parla vite d'autres choses et quand il mentionna que ses amis ingénieurs regrettaient qu'après quelques années passionnantes ils en étaient arrivés à ne faire pratiquement que de l'administration, j'en déduisis que la carrière d'ingénieur n'était pas faite pour moi car j'avais beaucoup trop de difficultés d'expression à l'époque, tant orales qu'écrites, pour choisir une voie où je risquais d'avoir à faire de l'administration. J'arrivai vite ce soir-là à la décision de faire de la recherche en Mathématiques. 
Assez naturellement, je demandai à Jacques-Louis Lions de me guider dans la recherche. Je sus assez vite que j'aurais un poste de Stagiaire de Recherche au CNRS à partir d'octobre 1968, après la fin de mon contrat de trois ans avec l'armée, mais peutêtre parce que le Gouvernement faisait un effort de développement pour la recherche à cette époque, je passai cette troisième année avec comme seule obligation de passer mon DEA. Jacques-Louis Lions mentionna plus tard la possibilité d'avoir un poste à l'IRIA, où il allait diriger un groupe de recherches en Analyse Numérique, mais le statut de cet institut n'était pas très clair à ce moment là. Les cours d'Analyse Numérique avaient lieu à l'Institut Blaise PASCAL dans le Nord de Paris, mais je suivais aussi des cours ainsi que le séminaire LIONS-SCHWARTz à l'IHP, ainsi que des cours et séminaires à l'IRIA.

Je ne connaissais alors l'Analyse Numérique qu'à travers ce que Jacques-Louis Lions enseignait, et il y mettait bien sûr un peu plus d'Analyse Fonctionnelle qu'il n'était vraiment nécessaire. Il m'avait cependant demandé d'apprendre le Fortran, ce que j'avais fait en trouvant un stage à IBM, mais je découvrais ensuite que le langage officiel du DEA était Algol ! J'écrivis quand même en Fortran le programme pour tester l'algorithme que Moïse Sibony m'avait demandé d'étudier pour mon stage de DEA. Je n'avais pas trouvé cet algorithme très performant $\mathrm{d} u$ point de vue théorique, et quand je me décidai à tester mon programme, j'eus la surprise de trouver l'Institut Blaise PASCAL fermé : la grève générale de mai 68 venait de commencer ! Je connaissais à Polytechnique une salle avec un ordinateur IBM, et comme la porte était ouverte et que personne n'était là quand j'y allais, je traitais mon paquet de cartes tout seul et comme mon programme tourna alors sans problème, je partis avec quelques pages de résultats sans que personne ne m'ait demandé ce que je faisais là ! Comme la suite montra que j'avais des tendances de théoricien, ce court programme fut le seul que j'écrivis en trente ans.

Pendant ces années d'initiation, j'apprenais de Jacques-Louis Lions comment transformer les équations aux dérivées partielles de la Mécanique des Milieux Continus ou de la Physique en problèmes variationnels, mais malgré ce que j'avais appris en Mécanique et en Physique à Polytechnique, je n'avais guère acquis d'intuition concernant le sens physique des équations que j'étudiais, à grand renfort de théorèmes généraux d'Analyse Fonctionnelle et d'espaces de Sobolev. Ce ne fut qu'après mes travaux avec François Murat, issus d'un sujet d'optimisation purement académique où nous avions redécouvert et étendu des résultats antérieurs de Sergio Spagnolo et d'Ennio De Giorgi, que je compris comment décrire les relations entre grandeurs microscopiques et macroscopiques à l'aide de diverses convergences faibles, ce qui me donnait enfin une plateforme plus mathématique pour initialiser l'immense tâche de donner des bases rationnelles pour ce qu'on m'avait enseigné dans mes cours de Physique (et mettre au rebut les arguments pseudo-probabilistes qu'on avançait alors comme explications). Si nos résultats sont maintenant connus sous le titre d'Homogénéisation, choisi par Ivo BABUŠKa, et souvent utilisés par certains qui se les approprient tout en montrant par leurs erreurs qu'ils ne les ont même pas bien compris, je tiens toujours à mentionner que c'était grâce aux travaux d'Henri SANCHEz-PALENCIA que nous avions compris pourquoi nos résultats abstraits avaient un rapport avec les questions de propriétés effectives de mélanges. 
Ceux de ma génération ont vu l'Analyse Numérique des équations aux dérivées partielles passer de l'enfance des années 60 où on pouvait certes déceler quelques excès d'Analyse Fonctionnelle, à la maturité des années 90 où on oublie souvent de compléter par des études théoriques les résultats de simulations numériques effectuées quelquefois sans trop y réfléchir, comme si on aimait à croire que la rapidité des ordinateurs allait corriger les erreurs de modélisation.

Il est bon de temps à autre de se pencher sur l'évolution d'une discipline pour analyser les facteurs qui l'ont fait évoluer, et on pourrait certainement apprendre beaucoup en étudiant les programmes des congrès annuels d'Analyse Numérique organisés depuis trente ans, mais je suis mal placé pour faire cette analyse, n'ayant essentiellement assisté qu'aux congrès où on m'avait invité, en 1977 à Imbours, en 1979 à Lamoura, et en 1982 à Belgodère, et je suis très reconnaissant à mes amis de Marseille d'avoir pensé à m 'inviter à nouveau, en 1998 à Arles. Je pense que c'est Jean CÉa qui avait organisé le premier de ces congrès il y a trente ans, alors qu'il enseignait encore à Rennes, et j'espère que lui et peut-être aussi les organisateurs des congrès suivants prendront leur plume pour nous rappeler comment les choses se passaient dans ces temps-là, où ces congrès ressemblaient beaucoup plus à des réunions de famille.

Isaac NEWTON (1642-1727) avait écrit "If I have seen a little further it is by standing on the shoulders of Giants", et je suis d'avis qu'on devrait suivre son exemple et penser de temps à autre à remercier ceux qui ont ouvert avant nous les voies qui nous permettent maintenant d'accéder à de nouvelles découvertes.

Je ne rappellerai pas l'oeuvre des pionniers de l'Analyse Fonctionnelle, au début du siècle, et je me bornerai comme d'habitude à citer surtout des mathématiciens que j'ai rencontrés personnellement, même si je ne peux prétendre avoir assez parlé avec eux pour qu'ils m'aient expliqué pourquoi et comment ils avaient introduit telle ou telle idée. Bien sûr, il faut au moins remonter aux années 30 pour citer les travaux de Sergei Sobolev et ceux de Jean LERAY, donnant le ton fondamental : s’intéresser aux équations aux dérivées partielles de la Mécanique des Milieux Continus et de la Physique et une fois identifiés les problèmes importants (ce qui n'est pas toujours si facile), créer de nouveaux outils mathématiques pour pouvoir enfin traiter ces problèmes correctement. Il faut cependant noter que si certains espaces de SoBoLev sont naturels pour un problème donné (car ils expriment en général qu'une énergie est finie), d'autres espaces, comme ceux qu'on utilise pour des questions de régularité de solutions, n'ont rien de naturel, même s’ils portent un nom célèbre, que ce soit celui de SoBolev ou un autre.

On m'a rapporté que Jean LeRAY avait conclu son exposé à un congrès en l'honneur de Jacques-Louis Lions par les mots "et c'est ainsi que SoBolev inventa les distributions". On ne m'a pas rapporté les réactions de Laurent SCHWARTz ce jour-là, mais malgré son refus de m'aider pour la défense de mes droits dans la lutte solitaire que j'ai mené contre le groupe des faussaires d'Orsay, je ne voudrais pas voler une seule idée à Laurent ScHWARTz pour l'attribuer à un autre ; je n'ai pas encore mené mon enquête sur la genèse de ces idées, et bien qu'un ami américain m'ait signalé qu'on devrait citer Herman WEYL pour la théorie des distributions, je n'ai pas encore pris le temps d'étudier cette piste. Les noms 
de Leray et de Schwartz apparaissent certainement dans ma liste des Géants.

Bien sûr, je mettrai aussi le nom de Jacques-Louis Lions dans cette liste. Son rôle a été capital dans le développement d'une école française d'Analyse Numérique des équations aux dérivées partielles, et dans la formation d'un certain nombre d'analystes appliqués, formés comme je l'ai été à l'utilisation des idées de l'Analyse Fonctionnelle et de la théorie des distributions pour certaines équations aux dérivées partielles issues de la Mécanique des Milieux Continus. On doit cependant se demander si son intérêt initial pour l'Analyse Numérique ne tenait pas à ce qu'il avait observé que dans les travaux de Peter LAX il y avait énormément d'Analyse Fonctionnelle associée à l'étude des schémas numériques ; la philosophie qu'un schéma linéaire est convergent si et seulement si il est stable et consistant avec l'équation aux dérivées partielles qu'il est censé approcher a dû lui permettre d'envisager un programme systématique de traduction de tous les schémas numériques déjà utilisés par les ingénieurs dans le langage de l'Analyse Fonctionnelle et le cadre des espaces de Sobolev.

Bien que Jacques-Louis Lions ait le plus souvent choisi ses problèmes en liaison avec des questions de Mécanique des Milieux Continus, ou plus généralement dans les Sciences de l'Ingénieur, je m'étais vite aperçu qu'il ne s`intéressait pas vraiment à la Mécanique, et que son but était finalement assez différent du mien. Pour expliquer certains de ses choix, je me demande finalement si son but n'était pas opposé au mien, car cela expliquerait pourquoi il avait à plusieurs reprises choisi soit d'attribuer mes résultats à d'autres (sans tenir compte de mes protestations), soit de ne pas mentionner mon nom quand il utilisait une des méthodes que j'avais développées. Je n'oublie pas l'accumulation de ses mérites, et le nom de Lions apparaît dans ma liste des Géants.

\section{Convergences faibles et Homogénéisation}

Au début des annés 70, j'ai développé avec François Murat les bases d'une théorie mathématique, qu'on appelle maintenant l'Homogénéisation, même si ce terme est souvent mal compris par beaucoup qui l'utilisent un peu n’importe comment.

Les premiers résultats mathématiques avaient été obtenus par Sergio Spagnolo, qui suivait des suggestions d'Ennio De Giongi. Sergio Spagnolo avait ainsi traité en 1967/68 des problèmes de diffusion, elliptiques ou paraboliques, dans le cas d'opérateurs symétriques, et il avait choisi le terme de G-convergence pour décrire ces résultats, pour rappeler qu'il s'agissait en fait de la convergence des fonctions de GreEN [53], [54].

François MURat avait obtenu en 1970 des résultats de non existence pour un problème d'optimisation purement académique, que Jacques-Louis Lions avait posé [41],[42]. Comme nous partagions un bureau à Jussieu à l'époque, j'avais été intrigué par ses ré-

sultats et nous avions ainsi commencé à travailler ensemble. Nous n'étions pas conscients que les idées que François utilisait pour ses contre-exemples étaient analogues à celles que Laurence C. Young avaient introduites à la fin des années 30 [67], et semblables à celles dont j'avais entendu parler au séminaire PALLu DE LA BARRIÈRE à l'IRIA (où les mesures de Young étaient appelées mesures paramétrées, et l'idée attribuée à GHoutla-Houri). Ce n'est que plus tard, parce que nous avions trouvé des démonstrations simples, que le lien avec des résultats antérieurs est apparu. Sans connaître les résultats antérieurs de 
Sergio Spagnolo, nous avons étudié le problème d'existence et défini une notion un peu différente, que François choisit plus tard d'appeler la H-convergence dans son cours à Alger [43], le H rappelant maintenant le terme Homogénéisation, introduit par Ivo BABUŠKa [1].

Puis nous avons découvert les travaux des Italiens, Tullio ZoLEzzi, l'article d'Ennio De Giorgi et Sergio Spagnolo [9] précurseur de la $\Gamma$-convergence, et celui d'Antonio Marino et Sergio Spagnolo [37], mais nous avions besoin de bornes plus précises dans notre travail et nous avons mis au point une première méthode pour obtenir des bornes sur les coefficients effectifs (les qualificatifs homogénéisés ou effectifs n'étaient pas encore utilisés), et j'en parlais à Rome en Avril 1974 [56] (en oubliant de mentionner François Murat, je crois). En passant en revue tous les cas où nous étions capables de calculer les limites, nous avons ensuite découvert le lemme Divergence-Rotationnel, précurseur de la Compacité par Compensation, et en Juin 1974 à Rocquencourt j'utilisais nos résultats pour déduire des conditions nécessaires d'optimalité (plus précises que celles que Jean CÉA utilisait avec son équipe à Nice), et je décrivais pour la première fois la convergence faible comme moyen de relier des grandeurs microscopiques et macroscopiques [57].

Nous avions compris que nos problèmes de convergences faibles étaient reliés aux questions de propriétés effectives des mélanges grâce aux travaux d'Henri SANCHEZPalencia, qui utilisait des développements asymptotiques pour des milieux périodiques $[50],[51]$.

Je passai l'année 1974/75 à Madison et je mis au point une méthode plus efficace que celle développée avec François Murat, basée sur l'utilisation systématique du lemme Divergence-Rotationnel (grâce aux explications de Joel RoBBIN je comprenais mieux la relation avec les formes différentielles), et je montrai cette méthode à Jacques-Louis Lions à un congrès à Marseille en Septembre 1975 [35], et il l'utilisa plus tard de manière systématique, appelant ma méthode "méthode de l'énergie" mais sans beaucoup me l'attribuer (certains Italiens l'appellent la "méthode de dualité", et je l'appelle moi même la "méthode des fonctions tests oscillantes"). Curieusement, Jacques-Louis Lions n'avait pas paru intéressé par nos résultats avant 1974, et il s'était mis à travailler pendant mon absence avec Alain Bensoussan et George Papanicolaou sur des problèmes avec structures périodiques parce qu'Ivo BABUŠKa l'avait convaincu de l'intérêt de cette question pour des problèmes d'ingénieur, mais il n'avait pas pensé à nous demander les détails de ce que j'avais écrit dans [57].

Du point de vue théorique, Leon SiMON découvrit indépendamment la même méthode : il connaissait les articles de Sergio Spagnolo, et avait fait travailler son élève MCConnell sur le sujet [38] et il mit ensuite lui-même la main à la pâte ; le referee, probablement de l'Ecole Italienne, lui signala ensuite mes travaux [52].

Olga OLEINIK découvrit la même méthode mais probablement pas indépendamment, car elle avait dû lire ou entendre en quoi consistait la méthode dans le cas périodique et elle l'étendit d'abord au cas presque périodique puis au cas général. Après ma lutte à Orsay, j'ai dû constater qu'on évitait systématiquement de citer mon nom et que certains s'attribuaient souvent mes idées. J'ai cru qu'Olga OLEINIK évitait de mentionner mon nom pour la même raison, mais elle a un jour expliqué qu'elle ignorait la référence à la H-convergence du cours d'Alger de François Murat [43]. J'en ai déduit que la théorie 
générale, sans hypothèse de périodicité, que j'avais développée avec François Murat et que j'avais exposée dans mon cours PECCOT au Printemps 1977, avait été passée sous silence.

Avant qu’il y ait une définition mathématique de ce que sont les coefficients effectifs d'un mélange en utilisant la G-convergence (SPagnolo et De Giorgi) ou la H-convergence (Murat et TARTar), il y avait eu pas mal de résultats formels, car on doit se rappeler que seuls les mathématiciens prennent le temps de définir les choses dont ils parlent. Parmi ces calculs formels, certains sont tout simplement faux, d'autres sont des conjectures qui ne sont pas toujours faciles à trancher, et d'autres sont exacts et se démontrent sans trop de difficultés une fois les définitions mathématiques précisées. Dans [1] Ivo BabušKa a mentionné des contributions anciennes, Poisson (1822), Maxwell (1873), RaYleigh (1892), et il a comparé les résultats de diverses formules anciennes proposées en Mécanique, Physique, Chimie. J'avais auparavant été très surpris de trouver dans un cours de Physique Théorique de LANDAU et LIFCHITZ un paragraphe où ils prétendaient calculer la conductivité d'un mélange en fonction des proportions des différents matériaux conducteurs utilisés, et j'en avais déduit qu'ils ne savaient pas de quoi ils parlaient : les calculs que j'avais faits avec François Murat montraient que, même en supposant le résultat isotrope, on ne pouvait pas déduire la H-limite en fonction des mesures de Young (ce qui est la manière mathématique de dire que les statistiques à un point sont insuffisantes). Notre première méthode pour obtenir des bornes sur les coefficients effectifs ne faisait que démontrer des inégalités "classiques" que nous ne connaissions pas à l'époque : "quand on dit qu'un résultat est bien connu, on veut dire qu'il est connu de ceux qui le connaissent bien", car il est souvent encore inconnu d'un autre groupe de personnes. Quand j'ai parlé à Rome en 1974, Sergio Spagnolo et Ennio De Giongi ignoraient ces bornes élémentaires que j'avais découvertes avec François Murat, et Ennio DE Giorgi était de la liste des Géants, et si je ne l'ai pas mentionné dans ma liste au début, c'est parce que je n'ai pas été influencé par ses idées. Nous n'avions pas redécouvert ces bornes car elles étaient inconnues des mathématiciens et personne ne nous avait signalé ces inégalités "bien connues", qui d'ailleurs ne pouvaient qu'être qualifiées de conjectures (puisqu'il fallait d'abord conjecturer qu'on pourrait définir correctement des coefficients effectifs et qu'ensuite certaines inégalités seraient vraies) ; Ennio De Giongi et Sergio Spagnolo auraient pu aisément déduire ces bornes de leur approche [9] si on les leur avait mentionnées (notre méthode était adaptée à la H-convergence, et donc n'était pas liée à un principe de minimisation comme dans le cas de la G-convergence).

En France, j'ai déjà cité Henri SANCHEz-PALENCIA comme ayant obtenu des résultats formels dans le cas périodique, et une fois la théorie générale développée, il n'a pas été difficile de vérifier qu’il avait eu raison sur certains problèmes, mais il y avait pas mal de difficultés pour d'autres, par exemple pour sa dérivation de la loi de DARCY à partir des équations de STOKEs stationnaires (ou Navier-Stokes stationnaires) dans un modèle périodique de milieu poreux : Jacques-Louis Lions m'avait signalé qu’il n'arrivait pas à appliquer ma méthode car dans ce problème elle requiert d'étendre la pression dans le solide avec des estimations précises, ce que j'ai ensuite fait pour une géométrie bidimensionnelle 
(fluide entourant le solide) et Grégoire Allaire l'a fait plus tard dans le cas (périodique) général. Il faut noter que MATHERon avait aussi obtenu la loi de DARCY en invoquant des principes probabilistes, mais j'ignore s'il existe une démonstration mathématique de ses résultats (la description des milieux poreux dans son approche était bien plus réaliste que la modélisation par un milieu périodique). Je ne pense pas que la Nature utilise des probabilités, et elles ne sont postulées à certains endroits que pour masquer notre incompréhension de ce qui s'y passe, et je préfère modéliser cette incertitude par des questions de convergences faibles adaptées, ce qui à première vue n'est pas trop différent puisqu'une espérance consiste à intégrer dans une variable cachée $\omega$, mais cela s'avère être très différent $\mathrm{du}$ point de vue philosophique, car mon approche permet de considérer des solutions d'équations aux dérivées partielles et de les laisser avoir toutes les oscillations possibles à condition qu'elles soient compatibles avec l'équation, et c'est difficile pour l'approche probabiliste qui postule trop les lois suivies (Joe KeLLER rapportait un jour qu'il y a assez longtemps il y avait pas mal d'articles sur la statistique des longueurs d'ondes des vagues à la surface de la mer, mais quand les satellites ont pû mesurer ce qui se passait dans la réalité, on s'est aperçu que toutes les lois proposées étaient fausses).

Aux Etats-Unis, j'avais appris (par Carl DeBoor) l'existence d'articles d'Tvo BABUŠKa, et j'avais ensuite bénéficié de ses conseils. Bien sûr, si on s'intéresse à l'Analyse Numérique des problèmes d'Homogénéisation (pour des structures périodiques) on doit consulter ses travaux [1],[2],[3],[4],[5],[39].

J'avais fait un exposé au Courant Institute à NYU, et Louis NIREnBerg m'avait montré des calculs de MCConnell (pour le cas des matériaux feuilletés en Elasticité linéarisée, étendant les calculs que François Murat avait fait pour le cas d'une équation de diffusion) [38]. Je n'avais pas rencontré Joe KelLer car, comme il me l'a dit peu après, il n'avait pas été intéressé par le titre de mon exposé (qui parlait de contrôle dans les coefficients). Il m'avait raconté qu'après avoir trouvé comment homogénéiser l'équation de la chaleur en une dimension d'espace, il avait demandé à Louis NIRENBERG s'il pouvait deviner comment on passait à la limite, et Louis NiREnBERg lui avait répondu qu'il fallait considérer la limite faible de l'inverse du coefficient de conductivité et, après cet effet de surprise, avait avoué qu'on lui avait expliqué ce résultat : il revenait de Pise et Sergio Spagnolo lui avait parlé de ses travaux. Joe KeLLER avait en fait déjà publié plusieurs articles sur des questions liées à 1'Homogénéisation [10],[27],[28],[29],[30],[31],[32],[40], [45],,[48].

J'avais aussi découvert un article de George PaPANICOLAOU oì j'avais trouvé une ressemblance avec ce que j'avais fait avec François Murat. Bien sûr, je n'ai jamais travaillé sur des modèles probabilistes, mais j'ai pu noter plus tard que ma méthode avait inspiré George Papanicolaou et Srinivasa Varadhan pour leur démonstration dans un cadre purement probabiliste [46].

J'ai appris plus tard qu'il y avait aussi eu des précurseurs en URSS, HRUSLOV dès les années 60 et BAHVALOV dans les années 70, mais une mention particulière doit être faite de K. LuR'IE. Indépendamment de ce que François Murat faisait aussi en 1970, mais en travaillant sur un problème d'optimisation réaliste, K. LUR'IE avait découvert un problème d'optimisation sans solution optimale, et s'il n'avait pas découvert l'Homogénéisation, 
il n'en était pas très loin, mais il avait suivi (consciemment) les idées de Pontryagin, alors que nous avions suivi (inconsciemment) les idées de L. C. Young : il avait trouvé comment écrire des conditions d'optimalité plus fortes que celles qu'on obtient en suivant l'idée habituelle due à HADAMARD (et ce n'était pas une extension évidente des résultats antérieurs), mais il avait seulement une intuition de ce que pouvaient être des solutions généralisées ; il l'avait appris en découvrant mon article [57]. C'est ce qu'il avait expliqué à Jean-Louis ARMAND quand celui-ci était venu le voir à Leningrad, ajoutant qu'il avait eu de la chance que [57] ait été publié pour un congrès portant sur le contrôle et non sur les équations aux dérivées partielles ; Jean-Louis ARMAND avait découvert quelques situations paradoxales dans les calculs que lui et d'autres ingénieurs faisaient pour trouver des formes optimales, et dans la littérature il n'avait trouvé que les travaux de LuR'IE qui semblaient en donner une explication, et c'est LuR'IE qui avait donc mentionné mes travaux à JeanLouis Armand, qui m'avait ensuite demandé de participer à un enseignement qu'il faisait. Malheureusement Robert KoHn (à qui j'avais expliqué mes travaux avec François MuRAT en juin 1980) semble avoir décidé à partir de 1983 de faire croire que lui et ses amis étaient les inventeurs des idées que j'avais développées, seul ou en collaboration avec François Murat, et en particulier il a essayé de faire croire que LuR'IE avait inventé la deuxième méthode pour obtenir des bornes sur les coefficients effectifs (basée sur la Compacité par Compensation que j'avais développée en collaboration avec François Murat en 1976/77), que j'avais exposée en 1977 [58] ; comme ce texte était en français, et que je n'en connaissais pas la référence exacte car on avait oublié de m'envoyer un exemplaire des comptes rendus de la conférence, je l'avais mentionnée en anglais dans un congrès à Minneapolis en 1984 (dont Kohn était un des organisateurs) [59]. Pendant un séjour au Courant Institute en juin 1980 , j'avais découvert comment choisir certaines fonctionnelles apparaissant dans ma méthode, et ayant signalé ces nouvelles bornes pour le cas isotrope, George PAPANICOLAOU m'avait dit de les comparer aux bornes de Hashin et Shtrikman [25].

C'était bien les mêmes bornes, et j'en avais donc donné la première démonstration, car la dérivation de leur formule par Hashin et SHTRIKMan ne tenait pas debout; par contre, leur construction de sphères concentriques pour montrer que ces bornes étaient atteintes était assez claire pour moi et je n'eus pas de mal à l'utiliser. Optimiste, François Murat suggéra de montrer que ma méthode donnerait aussi les bornes optimales pour le cas anisotrope ; il avait raison, mais il nous fallut un peu de temps pour faire les calculs pour une construction avec des ellipsoïdes confocaux (malgré les formules fournies par Edward FraenkeL), et j'exposai notre résultat en juin 1981 à un congrès à New York, mais sans rédiger ma conférence. J'enseignais ces résultats dans un cours d'option à l'Ecole Polytechnique l'année suivante et deux élèves, P. BRAIDY et D. Pouilloux, montrèrent qu'on pouvait arriver au même résultat sans la construction des ellipsoïdes confocaux, simplement en itérant la construction des matériaux feuilletés. Au printemps 1983 à Berkeley, je généralisai leur résultat en trouvant comment itérer avec des directions de feuilletage arbitraires, et j'expliquai ces résultats à Robert KoHn, et comme il devait aller à Leningrad voir LUR'IE il me demanda même l'autorisation de lui expliquer mes résultats exposés à New York deux ans avant, ce que je n'avais pas de raison de refuser à ce moment là. A l'automne, je rédigeai ces résultats pour une conférence dédiée à Ennio DE 
Gtorgi [60], mais LuR'IE les rédigea aussi, s'appropriant nos résultats que Robert KoHN lui avait expliqués, et Robert KoHN fait depuis référence au travail de LUR'IE comme s’il avait inventé ma méthode de [58]. Pour ne rien arranger, Graeme Milton (qui est honnête), avait baptisé ma méthode "méthode des translations" (ce qui ne m'avait pas paru un bon choix), et cela a permis à beaucoup de citer ma méthode sans me l'attribuer. Une démonstration des bornes de Hashin et SHTRIKMan en suivant leur méthode fut obtenue par la suite par D. TALBOT et John WiLlis [55] et par Robert Konn et Graeme Milton [33] dans le cas périodique ; il faut à ce sujet mentionner que les adeptes du cas périodique (qui peut-être ne savent pas utiliser les méthodes générales que j’ai développées avec François MuRat) écrivent souvent qu’il suffit de démontrer les bornes dans le cas périodique, et peut-être ne se rendent-ils pas compte que ce type de phrase n'a absolument aucun sens pour un lecteur auquel on n'a pas signalé qu’il existe une théorie générale n'utilisant pas la périodicité. Pourquoi d'ailleurs essayent-ils de cacher cette information aux lecteurs? En fait, on pourrait maintenant donner un sens à la dérivation de HasHiN et ShtrIKMan dans le cas général en utilisant les H-mesures que j'ai introduites en 1987/88; ma troisième méthode pour l'obtention de bornes (qui est basée sur l'utilisation des $\mathrm{H}-$ mesures), est d'une certaine manière une généralisation de leur idée, valable sans principe de minimisation [61].

Robert KoHN ayant agi de telle manière que beaucoup d'attributions postérieures à 1983 sont maintenant erronées, il a aussi essayé de brouiller les pistes des résultats anciens, au moins pour ceux qui ne lisent pas le français. Dans un livre (finalement édité avec Andrej Cherkaev), il a fait traduire des textes rédigés initialement en français et d'autres rédigés initialement en russe [8], mais il a tenu à attribuer [57] aussi à François Murat, ce que j'ai accepté, et François Murat, en contrepartie, a demandé que [43] me soit aussi attribué, et que [58] soit inclus, ce qui a été accepté. Robert KoHN savait-il que [58] était la référence à ma "méthode des translations" ? Dans l'introduction ma méthode est mentionnée comme méthode de Compacité par Compensation, mais elle ne m'est pas attribuée, et il n'est pas mentionné que l'article où elle est parue est traduit dans le livre ! Pourquoi d'ailleurs n'avoir pas traduit [60]?

Pourquoi insister sur ces questions de chronologie? Tout simplement parce que certains de ceux qui n'avaient pas participé à la création de ces idées mais voudraient maintenant faire croire qu'ils en sont spécialistes peuvent induire leurs lecteurs en erreur (j'ignore si c'est consciemment ou non). Attribuer à tort un résultat à quelqu'un qui n'y est pour rien n'est pas toujours très grave, mais il est plus important de noter que certains de leurs résultats sont incomplets ou peut-être même faux car ils ne dominent pas le sujet dont ils traitent. Il faut donc se méfier par exemple de ceux qui font des trous dans des matériaux sans s'occuper de la régularité du bord des trous, ou qui prétendent qu'on peut approcher un trou par un matériau peu conducteur ou peu élastique (ce qui est vrai) et en profitent pour intervertir deux limites sans le dire (ce qui est a moins douteux puisque les topologies qu'ils manipulent ne sont pas métrisables si on ne se restreint pas à des bornés). Si un auteur est prêt à attribuer à d'autres des résultats, pourquoi s'encombrerait-il de pareilles broutilles? 
En septembre 1995, à un congrès à l'Institute for Mathematics and its Applications à Minneapolis, quelqu'un qui par ses propres termes ne se considérait pas comme un mathématicien annonça qu'il avait démontré des bornes sur les coefficients effectifs en Elasticité linéarisée que les mathématiciens n'avaient pas obtenues; bien sûr, les mathématiciens sérieux connaissent les défauts de l'Elasticité linéarisée et ne passent pas trop de temps à ce jeu et cela aurait pû être vrai, mais ensuite il énonça un résultat faux, ce que je signalai suffisamment fort pour être entendu, mais personne ne réagit : il y avait deux erreurs dans ce qu’il faisait, et seul John WiLLIS est venu m'expliquer que le conférencier avait bien fait une erreur dans son utilisation du terme "non elliptique", et je lui ai expliqué plus tard où était la deuxième erreur. Cela ne dérangeait nullement les autres, qui peut-être propageront des erreurs analogues dans les articles qu'ils écriront.

Il faut savoir que la $\Gamma$-convergence, bien qu'elle généralise en principe la Gconvergence, n'est pas la même chose que l'Homogénéisation, terme qui devrait être compris comme la découverte des lois constitutives des matériaux hétérogènes et la description des oscillations qui sont créées dans les solutions par les oscillations des coefficients, sans faire d'hypothèse de périodicité, et aussi sans utiliser les conditions aux limites, car sinon on ne doit pas parler de propriétes effectives mais de comportement global du corps. Il est utile de signaler qu'il n'y a pas encore de théorie raisonnable de l'Homogénéisation pour l'Elasticité non linéaire, en dépit de ce qu'ont écrit certains qui sont tombés dans le piège de la $\Gamma$-convergence : on peut toujours minimiser une énergie pour des matériaux qui sont sortis depuis longtemps du domaine où leur réponse est élastique, mais on ne devrait pas parler d'Élasticité (cela arrive pour les alliages à mémoire de forme) ; on pourra consulter le livre de Ekhard SALJE par exemple [49], pour les définitions de termes comme coélastique, ferroïque, ferroélastique.

Peut-on encore admettre d'entendre parler de l'Elasticité sans jamais entendre parler de contraintes, et peut-on prendre au sérieux des gens qui croient encore que les matériaux élastiques passent leur temps à minimiser des énergies ? Quand on fait de l'Analyse Numérique, on cherche à être efficace, et si on veut résoudre $A(u)=0$ et qu'on connait plusieurs fonctions $F_{i}$ qui ont la propriété que $\operatorname{grad} F_{i}(u)=0$ implique $A(u)=0$, on a parfaitement le droit de minimiser l'une des fonctions $F_{i}$ pour approcher une solution de $A(u)=0$, et il importe peu que la fonction qu'on a choisie ait un sens physique ou non. On ne doit pas en déduire qu’il est utile de ne rien savoir sur la Mécanique des Milieux Continus et la Physique.

Y-a-t-il besoin de connaitre quoi que ce soit sur l'Homogénéisation quand on s`intéresse à l'Analyse Numérique ? Certainement, et je me souviens d'une question de James HyMan, à Los Alamos il y a une quinzaine d'années : dans un code multigrille il $\mathrm{y}$ avait à chaque niveau une valeur constante pour le coefficient de diffusion sur chaque maille, et l'idée était d'utiliser l'information venant des petites mailles pour améliorer la constante donnée sur une grande maille, et le code utilisait une formule pour faire ça, et il se demandait si on pouvait l'améliorer en utilisant l'Homogénéisation. Ce n'était pas exactement une question d'Homogénéisation, mais si un algorithme est robuste pour des questions d'oscillations sur des échelles arbitrairement petites, on devrait pouvoir l'utiliser 
pour des coefficients pas très réguliers et obtenir de bons résultats (en dimension 1 on utilise la moyenne de l'inverse de la conductivité), car il faut se rappeler que les estimations classiques d'erreurs ne sont valables que pour des coefficients assez réguliers. Les formules d'Homogénéisation de faible amplitude utilisant les H-mesures [61] devraient être utiles en Analyse Numérique (j’avais initialement développé les H-mesures pour obtenir des termes de correction car je m'étais aperçu qu'une formule de LANDAU et LIFCHITZ semblait donner de bons résultats, alors que sa dérivation était tout-à-fait farfelue).

Quand un mathématicien cherche à résoudre un problème, il essaie en général de résoudre une classe de problèmes, la difficulté étant de découvrir quels éléments sont importants dans le problème initial, mais il arrive souvent qu'après avoir mis au point une théorie générale, elle s'avère un peu lourde pour un problème donné et il est bon alors de mettre au point des méthodes intermédiaires qui perdent en généralité mais gagnent en souplesse.

Par exemple, il est vraiment rare qu'on ait besoin des mesures de Young, et je ne crois pas à l'utilité de les approcher. Pour le montrer, j'ai souvent utilisé l'exemple suivant : on veut minimiser la fonction coût $J(u)=\int_{0}^{T}\left(|y(t)|^{2}-|u(t)|^{2}\right) d t$ sachant que la commande $u$ est n'importe quelle fonction mesurable vérifiant $-1 \leq u(t) \leq 1$ presque partout et l'état $y$ est défini par l'équation d'état $y^{\prime}=u, y(0)=0$. On vérifie immédiatement que $J(u)>-T$ et que si une suite $u_{n}$ ne prend que les valeurs \pm 1 et converge faiblement vers 0 , alors $J\left(u_{n}\right)$ converge vers $-T$ (c'est Ivar EkELAND qui m'avait mentionné ce problème, et sa recette consistait à introduire je ne sais quel compactifié abstrait). On pourrait introduire les mesures de Young pour définir des solutions généralisées, mais les mesures de Young peuvent prédire la limite faible de n'importe quelle fonction d'une (sous-)suite $v_{n}$, alors qu'on s'aperçoit vite que seules les limites faibles $v$ et $w$ de $v_{n}$ et de $v_{n}^{2}$ sont nécessaires. Le problème relaxé qu'on obtient alors est convexe et consiste à minimiser la fonction cô̂t $\widetilde{J}(v, w)=\int_{0}^{T}\left(|y(t)|^{2}-w(t)\right) d t$ sachant que l'état $y$ est défini par l'équation d'état $y^{\prime}=v, y(0)=0$, et que la commande $(v, w)$ est n'importe quelle fonction mesurable prenant ses valeurs dans l'enveloppe convexe du morceau de parabole où $\left(u_{n}, u_{n}^{2}\right)$ prend ses valeurs, i.e. $-1 \leq v(t) \leq 1$ and $v^{2}(t) \leq w(t) \leq 1$ (d'après un résultat démontré avec François Murat en 1970, résultat dont Ivar EkELAND m’apprit ensuite qu’il était lié au théorème de LYAPUNOV, qui était caché dans la plupart des démonstrations qui se faisaient au séminaire PALLU DE LA BARRIÈRE) ; ce problème relaxé convexe a un seul minimum, $v=0, w=1$.

La même simplification a lieu pour des problèmes d'optimisation où l'Homogénéisation apparaît, car ayant développé des méthodes générales pour étudier cette question avec François Murat, nous avions passé pas mal de temps à comprendre comment obtenir des bornes optimales pour les coefficients effectifs (ce que nous avions fait pour les mélanges de deux conducteurs isotropes en 1981 [60]), avant de nous a percevoir que ces bornes n'étaient pas nécessaires dans certains cas [44], cette remarque ayant d'ailleurs déjà été faite auparavant par RAÏTum [47]. Dans [62], j’ai étendu cet argument au cas où on mélange des matériaux conducteurs qui peuvent être anisotropes, avec des tenseurs de conductivité (symétriques définis positifs) $M^{1}, \ldots, M^{m}$, dans des proportions locales arbitraires 
$\theta^{1}, \ldots, \theta^{m}$, c'est à dire ne vérifiant que les conditions $\theta^{1}, \ldots, \theta^{m} \geq 0, \theta^{1}+\ldots+\theta^{m}=1$ presque partout dans un ouvert $\Omega$ de $R^{N}$. Cela veut dire qu'on considère une suite de tenseurs de conductivité

$$
A_{n}=\sum_{k=1}^{m} \chi_{n}^{k}\left(R_{n}^{k}\right)^{T} M^{k} R_{n}^{k} \text { dans } \Omega,
$$

oì, pour chaque $n$, les $\chi_{n}^{k}, k=1, \ldots, m$, sont les fonctions caractéristiques d'ensembles mesurables disjoints, et tels que

$$
\chi_{n}^{k} \rightarrow \theta^{k} \text { dans } L^{\infty}(\Omega) \text { faible étoile, quand } n \rightarrow \infty, \text { pour } k=1, \ldots, m,
$$

et les $R_{n}^{k}$ sont des rotations (mesurables en $x$ ). On suppose que $A_{n}$ converge au sens de la H-convergence vers $A_{e f f}$, c'est-à-dire que

$$
\begin{gathered}
E_{n} \rightarrow E_{\infty} \text { dans } L_{l o c}^{2}\left(\Omega ; R^{N}\right) \text { faible, } \\
D_{n}=A_{n} E_{n} \rightarrow D_{\infty} \text { dans } L_{l o c}^{2}\left(\Omega ; R^{N}\right) \text { faible, } \\
\frac{\partial\left(E_{n}\right)_{i}}{\partial x_{j}}-\frac{\partial\left(E_{n}\right)_{j}}{\partial x_{i}} \text { reste dans un compact de } H_{l o c}^{-1}(\Omega) \text { fort, pour } i, j=1, \ldots, N, \\
\sum_{i=1}^{N} \frac{\partial\left(D_{n}\right)_{i}}{\partial x_{i}} \text { reste dans un compact de } H_{l o c}^{-1}(\Omega) \text { fort },
\end{gathered}
$$

impliquent $D_{\infty}=A_{e f f} E_{\infty}$ presque partout dans $\Omega$. Le problème difficile de l'Homogénéisation est de caractériser l'ensemble $\mathcal{K}\left(\theta^{1}, \ldots, \theta^{m} ; M^{1}, \ldots, M^{m}\right)$ de tous les $A_{\text {eff }}$ qui peuvent ainsi apparaître, et cet ensemble n'est pas connu en géneral sauf dans le cas $N=1$ (où on doit faire la moyenne harmonique des conductivités, car ce sont les résistivités qu'on doit ajouter pour des conducteurs en série), et dans le cas d'un mélange de deux conducteurs isotropes obtenu avec François Murat en 1981 [60]. Fort heureusement, il n'est pas toujours nécessaire de connaitre cet ensemble $\mathcal{K}$, et on peut souvent se contenter pour un vecteur $E$ donné de caractériser toutes les valeurs possibles de $D=A_{e f f} E$ quand $A_{e f f}$ parcourt $\mathcal{K}$, et la caractérisation de cette projection est

$$
\begin{gathered}
\left\{A_{e f f} E: A_{e f f} \in \mathcal{K}\left(\theta^{1}, \ldots, \theta^{m} ; M^{1}, \ldots, M^{m}\right)\right\}=\text { boule de diamètre }\left[\lambda_{-} E, \lambda_{+} E\right] \\
\frac{1}{\lambda_{-}}=\sum_{k=1}^{m} \frac{\theta^{k}}{\lambda_{1}\left(M^{k}\right)} \\
\lambda_{+}=\sum_{k=1}^{m} \theta^{k} \lambda_{N}\left(M^{k}\right)
\end{gathered}
$$

où $\lambda_{1}(M)$ et $\lambda_{N}(M)$ désignent respectivement la plus petite et la plus grande valeur propre de $M$. Si on dénote par $\mathcal{B}\left(\theta^{1}, \ldots, \theta^{m} ; M^{1}, \ldots, M^{m}\right)$ l'ensemble des matrices symétriques $M$ satisfaisant $\lambda_{-} \leq \lambda_{1}(M) \leq \lambda_{N}(M) \leq \lambda_{+}$, alors on a

$$
\mathcal{K}\left(\theta^{1}, \ldots, \theta^{m} ; M^{1}, \ldots, M^{m}\right) \subset \mathcal{B}\left(\theta^{1}, \ldots, \theta^{m} ; M^{1}, \ldots, M^{m}\right),
$$


$\left\{A_{e f f} E: A_{e f f} \in \mathcal{K}\left(\theta^{1}, \ldots, \theta^{m} ; M^{1}, \ldots, M^{m}\right)\right\}=\left\{M E: M \in \mathcal{B}\left(\theta^{1}, \ldots, \theta^{m} ; M^{1}, \ldots, M^{m}\right)\right\}$, $\forall E \in R^{N}$, mais $\mathcal{K} \neq \mathcal{B}$ ( $\mathcal{B}$ est convexe, mais il y a des cas où $\mathcal{K}$ n'est pas convexe).

Bien que les défauts de l'Elasticité linéarisée soient bien connus, il n'en reste pas moins que beaucoup de problèmes d'optimisation de forme ont trait à des questions d'Elasticité, et comme on sait en fait très peu de choses en Elasticité non linéaire, il serait quand même utile de connaitre un résultat analogue pour l'Elasticité linéarisée.

En fait il serait utile de développer ce type de résultats de manière plus générale. Par exemple, la difficulté fondamentale pour les écoulements turbulents vient du fait que la classe des opérateurs de transport du premier ordre n'est pas stable par Homogénéisation ; on sait trouver les équations effectives dans certains cas, et cela fait apparaître des termes non locaux, et même sans caractériser la classe exacte des équations effectives, on pourrait peut-être obtenir des équations simplifiées ayant les mêmes solutions ou des solutions assez proches.

\section{H-mesures et variantes}

J'ai introduit les H-mesures en 1987/88 d'abord pour des questions d'Homogénéisation de faible amplitude (d'où mon choix du préfixe $H$ ), puis je les ai utilisées pour obtenir des bornes sur les coefficients effectifs et enfin pour démontrer des théorèmes de propagation d'oscillations (et d'effets de concentration) pour des systèmes hyperboliques (où $x$ et $t$ jouent le même rôle), mais je n'ai pas réussi à les utiliser pour obtenir des résultats de compacité par moyenne [61]. Patrick GÉRARD a introduit les mêmes objets indépendamment (dans un cadre plus général car il considérait des fonctions à valeurs dans des espaces de HiLbert alors que je ne travaillais qu'en dimension finie), et il les a appelées "mesures de défaut microlocales" car il avait été formé à l'école de Lars Hörmander où on s’intéresse à la régularité microlocale de solutions d'équations aux dérivées partielles à coefficients réguliers (et où malheureusement on appelle propagation de singularités ce qui est en fait de la propagation de régularité microlocale), et il a introduit ces mesures précisément pour démontrer des résultats de compacité par moyenne [13].

Il n'est pas étonnant que ceux qui veulent éviter de m'attribuer mes idées utilisent le terme de mesures de défaut microlocales même pour parler de résultats de propagation d'oscillations (et d'effets de concentration) que j'avais été le premier à introduire correctement, mais heureusement Patrick GÉRARD est honnête. Sa formation ne l'avait pas préparé à avoir le même point de vue que moi concernant les applications de ces mesures, et c'est ce qui lui a fait écrire qu'on ne peut pas définir ces mesures microlocales sur les variétés et qu'elles sont seulement définies à une multiplication par une fonction près, mais que ce n'est pas grave car seul le support de ces mesures est important : c'était naturel pour une personne familière avec les propriétés du support singulier essentiel d'une distribution introduit par Lars Hörmander [26], car ce n'est qu'un objet géométrique qui est une région interdite, et il ne pensait pas qu'il était important d'y définir quelque chose. Certains adeptes de la théorie de Lars HörmANDER prétendent à tort démontrer des résultats de propagation de singularités car ils montrent seulement que le support singulier essentiel est une union de bicaractéristiques, et même avec des améliorations considérant la régularité 
$H^{s}$ microlocale, ils n'ont toujours que des objets géométriques et ils ne peuvent pas dire s’il y a ou non un échange d'informations entre deux rayons passant au même point au même instant.

Certains diront que l'Optique Géométrique n'est pas une théorie formelle car certains calculs peuvent être faits avec précision : on cherche une solution de la forme $A e^{i \varphi}$, où l'amplitude $A$ et la phase $\varphi$ dépendent d'un paramètre de fréquence $\nu$ et on essaye d'obtenir ainsi une solution de l'équation des ondes quand la fréquence tend vers l'infini, et on trouve que la phase doit satisfaire l'équation eikonale (qui est une équation de HamiLTonJACOBI et présente des difficultés le long des caustiques) et que l'amplitude doit satisfaire une équation de transport qui a besoin de $\operatorname{grad} \varphi$, et comme on peut dire des choses précises sur ces équations on peut évidemment faire correctement l'analyse qu'il existe des solutions de l'équation des ondes qui ressemblent à ça et dont l'énergie est transportée le long des bicaractéristiques, les rayons lumineux. C'est une petite vérité enveloppée dans un gros mensonge (l'arme habituelle de la propagande), car le problème est de savoir si les solutions oscillantes de l'équation des ondes qu'on ne fabrique pas soi-même ont les mêmes propriétés. Qu'est-ce-qui empêche d'autres solutions oscillantes de l'équation des ondes de se comporter différemment? La Théorie Géométrique de la Diffraction de Joe KeLler va plus loin que l'Optique Géométrique, puisqu'elle traite par exemple des rayons rasants qui perdent leur intérêt si la fréquence devient trop grande, mais Joe KeLLER a signalé lui-même qu'elle donne des résultats faux sur les caustiques car elle prévoit une amplitude infinie.

Les H-mesures permettent de montrer que toutes les solutions oscillantes (j'utilise ce terme comme incluant les effets de concentration) de l'équation des ondes suivent les règles de l'Optique Géométrique, et cela pourra paraitre paradoxal quand on sait que les H-mesures n'ont pas de longueur caractéristique et donc ne peuvent pas voir la fréquence et n'ont pas la possibilité de déterminer la phase (alors que grad $\varphi$ intervient explicitement dans l'équation de transport pour l'amplitude). Il pourra paraître paradoxal aussi que les H-mesures utilisent seulement l'idée de ce que sont les opérateurs pseudo-différentiels, que Joseph Kohn et Louis NiRENBERG avaient introduit initialement pour des problèmes de nature elliptique [34], et non les opérateurs Fourien intégraux que Lars Hörmander avait introduits justement pour pouvoir traiter des situations hyperboliques [26] (ma construction ressemble un peu à celle $d u$ support singulier essentiel). Ce sont des remarques parfaitement valables, qui montrent qu'il est important de rester ouvert à des points de vue nouveaux quand on fait de la recherche, et qu'il est utile de se demander d'où viennent les idées qu'on utilise : l'idée des H-mesures était très naturelle pour certaines questions d'Homogénéisation et après avoir entrevu leur utilité dans une formule obtenue en 1984 [59], j'avais trouvé la motivation en 1986 pour expliquer pourquoi une dérivation très farfelue de LANDAU et LifCHITz donnait de bons résultats, mais je n'ai trouvé qu'en 1987 le maillon manquant dans ma démonstration d'existence ; j'ai ensuite décidé d'étudier la propagation des oscillations pour une équation du premier ordre pour voir si elle avait lieu le long des mêmes bicaractéristiques qui servaient à propager la régularité microlocale, et ma démonstration de 1988 s'appliquait aisément à d'autres équations linéaires ayant une loi de conservation sesquilinéaire pour leurs solutions complexes, l'équation des ondes, de 
Maxwell, de Dirac, de l'Elasticité linéarisée.

Si j'avais pris les problèmes dans l'ordre inverse, je ne serais probablement arrivé à rien : la raison pour laquelle on arrive à se passer de la phase (pour la limite de l'Optique Géométrique) est que les H-mesures peuvent décrire l'énergie se propageant dans une direction $\xi$ (à la vitesse $c$ par exemple), et comme ce sont des mesures en $(x, t)$ et sa variable duale $(\xi, \tau)$ normalisée à la norme 1 (et ces mesures vivent sur l'ensemble où $|\tau|=c|\xi|$ ), l'équation qu'on découvre pour la H-mesure $\mu$ est une équation aux dérivées partielles du premier ordre dans toutes les variables; il n'y a aucune difficulté à avoir plusieurs ondes planes oscillant à des fréquences grandes (mais pas forcément des fréquences voisines) car elles correspondent à des $\xi$ différents et il ne peut pas y avoir d'échange d'informations entre ces ondes quand elles passent en même temps en un point puisque les informations sont à des endroits différents en $(x, t, \xi)$.

Je voudrais essayer de décrire, comme je l'avais déjà fait dans [66], quelques idées concernant l'Analyse Numérique, et je pense que les considérations qui précèdent sont utiles pour bien montrer pourquoi il est important de penser à des formulations nouvelles. Je ne veux pas répéter ici toutes les définitions, qu'on peut consulter dans [61],[63],[64],[65],[66] par exemple, et qu'on peut comparer aux idées de Patrick GÉRARD [6],[13],.., [21], [24], ou à l'idée de Pierre-Louis Lions et T. Paul [36]. A force de répéter que j'aimerais qu'on m'attribue mes résultats, il est arrivé qu'on m'attribue une démonstration utilisant des termes que je ne connaissais pas, et dont Patrick GÉRARD devait être l'auteur, et il doit être clair que j'aimerais qu'on attribue à chacun ses résultats (nous avons collaboré sans avoir écrit d'article en commun, et j'ai moi-même signalé certaines de nos remarques dans $[66])$.

Les problèmes de transport d'oscillations dans les systèmes hyperboliques ont une certaine importance pratique, et il est bon de connaitre les outils théoriques ou tout au moins d'en avoir une certaine idée, mais j'ai peut-être brossé un tableau trop idyllique de la situation, car il y a encore beaucoup de choses à améliorer.

Par exemple les coefficients des équations doivent être de classe $C^{1}$ pour obtenir l'équation de transport satisfaite par les H-mesures; à part dans des situations académiques comme la réflection sur un bord plat traitée dans [64], je ne sais pas étudier les problèmes de réflection ou de réfraction aux interfaces ; comme la Théorie Géométrique de la Diffraction de Joe KelLer prévoit une forte dépendance par rapport à la fréquence pour les rayons rasants, on doit donc certainement utiliser une approche différente en utilisant des variantes de H-mesures prenant en compte des longueurs caractéristiques (comme les mesures semi-classiques introduites par Patrick GÉRARD).

A ce sujet, il faut noter que la théorie de Joe KELLER prévoit que les rayons rasants suivent les géodésiques $\mathrm{du}$ bord et qu'il y a un coefficient d'atténuation exponentiel où la racine cubique du nombre d'onde intervient; il y a une idée de Patrick GÉRARD pour traiter cette propagation le long du bord dans ses travaux avec E. Leichtnam [22],[23] (pour les mesures semi-classiques et non pour les H-mesures, puisque la fréquence joue un rôle important).

La prise en compte des conditions initiales (et des conditions aux limites) doit être 
améliorée. J'avais traité le cas d'une équation scalaire du premier ordre [61], mais la situation est plus compliquée en général, et le cas de l'équation des ondes a été traité par Gilles FrancFort et François Murat [11] (avec les conseils techniques de Patrick GÉrard). Le cas de l'Elasticité linéarisée pose cependant un problème plus difficile, et il est important pour les applications (on pourra consulter les comptes rendus d'une conférence édités par Guy Chavent, George Papanicolaou, P. Sacks et Bill Symes [7], pour apprendre à quoi servent ces études).

Il semble important de penser à l'approximation numérique de mesures portées par des variétés. Dans [61], j'ai défini les H-mesures sur un ouvert $\Omega$ de $R^{N}$ comme étant des mesures de RADON sur $\Omega \times S^{N-1}$, mais en fait $S^{N-1}$ devrait être remplacé par un quotient. Bien qu'assez semblable à la définition de Patrick GÉRARD des mesures semi-classiques, mon approche pour les variantes à une longueur caractéristique exposée sur un exemple dans [64] est plus difficile à manipuler parce que le choix de $S^{N-1}$ n'est pas très bon, et le choix de la représentation n'est donc pas tout à fait anodin. Ce choix pourrait devenir bien plus crucial pour les variantes à plusieurs longueurs caractéristiques, pour des situations hiérarchiques comme je l'ai mentionné dans [64]. Comme pour le cas des mesures de Young où on a rarement besoin d'approcher les mesures de Young complètes, les $\mathrm{H}-$ mesures interviennent souvent par certains de leurs moments, et on pourrait se restreindre à la description de ces moments ; les problèmes qu'on obtient alors ne sont pas toujours simples, comme dans l'étude que j'ai faite avec Gilles FrancForT et François Murat des moments d'ordre 4 d'une mesure positive sur la sphère $S^{2}$, en liaison avec des problèmes d'Elasticité linéarisée [12].

Il y a des problèmes où interviennent à la fois des mesures de Young et des Hmesures, comme les problèmes de micromagnétisme que j'avais exposés dans [63],[65], mais parce que je m'intéressais seulement au cas où le terme d'échange disparaissait à la limite, j'avais utilisé des résultats (non publiés) obtenus avec François Murat concernant les relations entre mesures de Young et H-mesures pour déduire un problème relaxé utilisant seulement les mesures de Young, et même me ramener au cas d'un problème convexe. Il y a d'autres problèmes où une meilleure compréhension de ces relations semble nécessaire.

\section{Conclusions.}

J'ai essayé de retracer une partie du chemin parcouru en trente ans. De l'Homogénéisation dont j'ai décrit les origines, de la Compacité par Compensation dont je n'ai pas parlé mais où j'aurais aussi pu décrire quelques erreurs propagées par des gens qui ont peut-être consciemment essayé d'induire les débutants en erreur, des H-mesures dont j'ai fait aussi un survol trop rapide, rien de tout cela n'était enseigné quand je passais mon DEA d'Analyse Numérique en 1968.

J'avais suivi à l'Ecole Polytechnique un cours d'Architecture, qui consistait en une projection de diapositives que le professeur commentait (il s'appelait ARSAC, je crois) ; je me souviens qu'un jour il avait énoncé que les grandes oeuvres architecturales prenaient dix ans à construire, mais je ne me souviens pas de la liste de ces oeuvres qu'il nous 
avait montrées à l'appui de sa thèse. J'ai enseigné l'Homogénéisation et la Compacité par Compensation en $1977 / 78$, et j'ai parlé de H-mesures à partir de 1988 , mais les idées que j'ai essayé de développer ensuite n'étaient pas prêtes pour 1998.

Il était clair pour moi que pendant que je développais l'Homogénéisation, la Compacité par Compensation, les H-mesures, j'aidais les mathématiciens à mieux comprendre la Mécanique des Milieux Continus et la Physique, mais je me suis aperçu que l’inertie est beaucoup plus grande que ce que j’imaginais. Je pensais aussi que cela devait déboucher sur des méthodes plus efficaces pour l'approximation des équations aux dérivées partielles de la Mécanique des Milieux Continus et la Physique.

\section{Remerciements.}

De 1982 à 1987, ma recherche a été effectuée au Commissariat à l'Energie Atomique à Limeil, grâce à l'aide de Robert DautraY, que je tiens à remercier. Depuis 1987, ma recherche a été effectuée à CARNEgIE MELLon University à Pittsburgh, avec un soutien de la National Science Foundation: contrats DMS-8803317, DMS-9100834, DMS-94-01310, DMS-97-04762, et par l'intermédiaire d'un contrat au Center for Nonlinear Analysis. Je tiens aussi à remercier le Max PLANCK Institute for Mathematics in the Sciences à Leipzig pour son soutien pendant mon année sabbatique 1997/98.

\section{Références}

[1] I. BABUŠKA "Homogenization and its application. Mathematical and computational problems," Numerical Solution of Partial Differential Equations, III, (Proc. Third Sympos. (SYNSPADE), Univ. Maryland, College Park, Md., 1975), pp. 89-116, Academic Press, New York, 1976.

[2] I. BABUŠKA "Homogenization approach in engineering," Computing methods in applied sciences and engineering (Second Internat. Sympos., Versailles, 1975), Part 1, pp. 137-153, Lecture Notes in Econom. and Math. Systems, Vol. 134, Springer, Berlin, 1976.

[3] I. BABUšKa "The computational aspects of the homogenization problem," Computing methods in applied sciences and engineering (Proc. Third Internat. Sympos., Versailles, 1977), I, pp. 309-316, Lecture Notes in Math., 704, Springer, Berlin, 1979.

[4] I. BabušKa "Solution of interface problems by homogenization, I, II, III" SIAM J. Math. Anal. 7 (1976), no. 5, 603-634; SIAM J. Math. Anal. 7 (1976), no. 5, 635-645; SIAM J. Math. Anal. 8 (1977), no. 6, 923-937.

[5] I. Babuška, R. C. Morgan "Composites with a periodic structure: mathematical analysis and numerical treatment,"

[6] N. Burco, P. GÉrard "Condition nécessaire et suffisante pour la contrôlabilité exacte des ondes," C. R. Acad. Sci. Paris Sér. I Math. 325 (1997), no. 7, 749-752. 
[7] Inverse problems in wave propagation. Papers from the workshop held at the University of Minnesota, Minneapolis, MN, March 6-17, 1995. Edited by Guy Chavent, George Papanicolaou, Paul Sacks, William W. Symes. The IMA Volumes in Mathematics and its Applications, 90. Springer-Verlag, New York, 1997.

[8] Topics in the mathematical modelling of composite materials. Progress in Nonlinear Differential Equations and their Applications, 31, A. CHERKAEv . R. KoHN eds., Birkhäuser Boston, Inc., Boston, MA, 1997.

[9] E. De Giorgi, S. Spagnolo "Sulla convergenza degli integrali dell'energia per operatori ellitici del 2 ordine," Boll. Un. Mat. Ital. (4) 8 (1973), 391-411.

[10] J. E. Flaherty, J. B. Keller "Elastic behavior of composite media," Comm. Pure Appl. Math. 26 (1973), 565-580.

[11] G. A. Francfort, F. Murat "Oscillations and energy densities in the wave equation," Comm. Partial Differential Equations 17 (1992), no. 11-12, 1785-1865.

[12] G. Francfort, F. Murat, L. Tartar "Fourth-order moments of nonnegative measures on $S^{2}$ and applications." Arch. Rational Mech. Anal. 131 (1995), no. 4, 305-333.

[13] P. GÉRard "Compacité par compensation et régularité 2-microlocale," Séminaire sur les équations aux Dérivées Partielles, 1988-1989, Exp. No. VI, 18 pp., Ecole Polytech., Palaiseau, 1989.

[14] P. GÉrard "Moyennisation et régularité deux-microlocale," Ann. Sci. Ecole Norm. Sup. (4) 23 (1990), no. 1, 89-121.

[15] P. Gérard "Microlocal defect measures," Comm. Partial Differential Equations 16 (1991), no. $11,1761-1794$.

[16] P. GÉrard "Mesures semi-classiques et ondes de Bloch," Séminaire sur les Equations aux Dérivées Partielles, 1990-1991, Exp. No. XVI, 19 pp., Ecole Polytech., Palaiseau, 1991.

[17] P. GÉRARD "Remarques sur l'analyse semi-classique de l'équation de Schrödinger non linéaire," Séminaire sur les Equations aux Dérivées Partielles, 1992-1993, Exp. No. XIII, 13 pp., Ecole Polytech., Palaiseau, 1993.

[18] P. GÉRARD "Microlocal analysis of compactness," Nonlinear partial differential equations and their applications. Collège de France Seminar, Vol. XII (Paris, 1991-1993), pp. 75-86, Pitman Res. Notes Math. Ser., 302, Longman Sci. Tech., Harlow, 1994.

[19] P. GÉRARD "Injections de Sobolev critiques, mesures microlocales et ondes non linéaires," Séminaire sur les Equations aux Dérivées Partielles, 1994-1995, Exp. No. II, 13 pp., Ecole Polytech., Palaiseau, 1995.

[20] P. GÉRARD "A microlocal version of concentration-compactness," Partial differential equations and mathematical physics (Copenhagen, 1995; Lund, 1995), pp. 143-157, Progr. Nonlinear Differential Equations Appl., 21, Birkhäuser Boston, Boston, MA, 1996.

[21] P. GÉRARD "Oscillations and concentration effects in semilinear dispersive wave equations," J. Funct. Anal. 141 (1996), no. 1, 60-98.

[22] P. GÉrard, E. Leichtnam "Ergodicité de fonctions propres pour des problèmes aux limites," Séminaire sur les Equations aux Dérivées Partielles, 1991-1992, Exp. No. XIV, 14 pp., Ecole Polytech., Palaiseau, 1992. 
[23] P. GÉrard, E. Leichtnam "Ergodic properties of eigenfunctions for the Dirichlet problem," Duke Math. J. 71 (1993), no. 2, 559-607.

[24] P. Gérard, P. A. Markowich, N. J. Mauser, F. Poupaud "Homogenization limits and Wigner transforms," Comm. Pure Appl. Math. 50 (1997), no. 4, 323-379.

[25] Z. Hashin, S. Shtrikman "A variational approach to the theory of effective magnetic permeability of multiphase materials," J. Applied Phys. 33, (1962), pp. 3125-3131.

[26] L. Hörmander The analysis of linear partial differential operators. I. Distribution theory and Fourier analysis, II. Differential operators with constant coefficients, III. Pseudodifferential operators, IV. Fourier integral operators. Grundlehren der Mathematischen Wissenschaften, 256, 257, 274, 275. Springer-Verlag, Berlin-New York, 1983, 1983, 1985, 1985.

[27] F. C. Karal, J. B. Keller "Elastic, electromagnetic, and other waves in a random medium," J. Mathematical Phys. 5, 1964, 537-547.

[28] J. B. Keller "Wave propagation in random media," 1962 Proc. Sympos. Appl. Math., Vol. XIII pp. 227-246, American Mathematical Society, Providence, R.I.

[29] J. B. KELLER "A theorem on the conductivity of a composite medium," J. Mathematical Phys. 5 1964, 548-549.

[30] J. B. Kellen "Stochastic equations and wave propagation in random media," 1964 Proc. Sympos. Appl. Math, Vol. XVI pp. 145-170, Amer. Math. Soc., Providence, R.I.

[31] J. B. KelLer, O. FARouk "Partial differential equations with periodic coefficients and Bloch waves in crystals," J. Mathematical Phys. 5, 1964, 1499-1504.

[32] J. B. Keller, F. C. Karal "Effective dielectric constant, permeability, and conductivity of a random medium and the velocity and attenuation coefficient of coherent waves," $J$. Mathematical Phys. 7, 1966, 661-670.

[33] R. V. Kohn, G. W. Milton "On bounding the effective conductivity of anisotropic composites," Homogenization and effective moduli of materials and media (Minneapolis, Minn., 1984/1985), pp. 97-125, IMA Vol. Math. Appl., 1, Springer, New York-Berlin, 1986.

[34] J. J. Kohn, L. Nirenberg "An algebra of pseudo-differential operators," Comm. Pure Appl. Math. 18 (1965), 269-305.

[35] J.-L. Lions "Asymptotic behaviour of solutions of variational inequalities with highly oscillating coefficients," Applications of methods of functional analysis to problems in mechanics (Joint Sympos., IUTAM/IMU, Marseille, 1975), pp. 30-55. Lecture Notes in Math. $\mathbf{5 0 3 .}$ Springer, Berlin, 1976.

[36] P.-L. Lions, T. Paul "Sur les mesures de Wigner," Rev. Mat. Iberoamericana 9 (1993), no. $3,553-618$.

[37] A. Marino, S. Spagnolo "Un tipo di approssimazione dell'operator $\sum_{i j} D_{i}\left(a_{i j}(x) D_{j}\right)$ con operatori $\sum_{j} D_{j}\left(\beta(x) D_{j}\right), "$ Ann. Scuola Norm. Sup. Pisa (3) 23 (1969), 657-673.

[38] W. H. MCCONNELL "On the approximation of elliptic operators with discontinuous coefficients," Ann. Scuola Norm. Sup. Pisa Cl. Sci. (4) 3 (1976), no. 1, 121-137.

[39] R. C. Morgan, I. Babuška "An approach for constructing families of homogenized equations for periodic media. I, An integral representation and its consequences, II, Properties of the kernel," SIAM J. Math. Anal. 22 (1991), no. 1, 1-15; SIAM J. Math. Anal. 22 (1991), no. $1,16-33$. 
[40] J. A. Morrison, G. Papanicolaou, J. B. Keller "Mean power transmission through a slab of random medium," Comm. Pure Appl. Math. 241971 473-489.

[41] F. Murat "Un contre-exemple pour le problème du contrôle dans les coefficients," $C$. $R$. Acad. Sci. Paris Sér. A-B 273 1971, A708-A711.

[42] F. Murat "Théorèmes de non-existence pour des problèmes de contrôle dans les coefficients," C. R. Acad. Sci. Paris Sér. A-B 274 (1972), A395-A398.

[43] F. Murat "H-convergence," Séminaire d'analyse fonctionnelle et numérique, Université d'Alger, 1977-78.

[44] F. Murat, L. Tartar "Calcul des variations et homogénéisation," Les méthodes de l'homogénéisation: théorie et applications en physique, (Bréau-sans-Nappe, 1983), pp. 319369, Collect. Dir. Etudes Rech. Elec. France, 57, Eyrolles, Paris, 1985.

[45] G. Papanicolaou, J. B. Keller "Stochastic differential equations with applications to random harmonic oscillators and wave propagation in random media," SIAM J. Appl. Math. 21 (1971), 287-305.

[46] G. Papanicolaou, S. R. S. Varadhan "Diffusion in regions with many small holes," Stochastic differential systems (Proc. IFIP-WG 7/1 Working Conf., Vilnius, 1978), pp. 190206, Lecture Notes in Control and Information Sci. 25 Springer, Berlin-New York, 1980.

[47] U. E. Raїтum "Extension of extremal problems connected with linear elliptic equations," Dokl. Akad. Nauk SSSR 243 (1978), no. 2, 281-283. Soviet Math. Dokl. 19 (1978), no. 6, 1342 - 1345 (1979).

[48] L. A. Rubenfeld, J. B. Keller "Bounds on elastic moduli of composite media," SIAM J. Appl. Math. 17, 1969, 495-510.

[49] E. K. H. SalJe Phase Transitions in Ferroelastic and Co-elastic Crystals. 1993, Cambridge University Press, Cambridge, UK.

[50] E. SancheZ-Palencia "Solutions périodiques par rapport aux variables d'espaces et applications," C. R. Acad. Sci. Paris, Sér. A-B 271 (1970), A1129-A1132.

[51] E. Sanchez-Palencia "Equations aux dérivées partielles dans un type de milieux hétérogènes," C. R. Acad. Sci. Paris, Sér. A-B 272 (1972), A395-A398.

[52] L. Simon "On G-convergence of elliptic operators," Indiana Univ. Math. J., 28 (1979), $587-594$.

[53] S. Spagnolo "Sul limite delle soluzioni di problemi di Cauchy relativi all' equazione del calore," Ann. Scuola Norm. Sup. Pisa, (3) 21 (1967), 657-699.

[54] S. Spagnolo "Sulla convergenza di soluzioni di equazioni paraboliche ed ellitiche," Ann. Scuola Norm. Sup. Pisa (3) 22 (1968), 571-597.

[55] D. R. S. Talbot, J. R. Willis "Variational principles for inhomogeneous nonlinear media," IMA J. Appl. Math. 35 (1985), no. 1, 39-54.

[56] L. Tartar "Convergence d'opérateurs différentiels," Atti Giorni Analisi Convessa e Applicazioni (Roma, 1974), 101-104. 
[57] L. TaRTaR "Problèmes de contrôle des coefficients dans des équations aux dérivées partielles," Control theory, numerical methods and computer systems modelling (Internat. Sympos., IRIA LABORIA, Rocquencourt, 1974) pp. 420-426. Lecture Notes in Econom. and Math. Systems, Vol. 107, Springer, Berlin, 1975.

[58] L. TARTAR "Estimations de coefficients homogénéisés," Computing methods in applied sciences and engineering (Proc. Third Internat. Sympos., Versailles, 1977), I, pp. 364-373, Lecture Notes in Math., 704, Springer, Berlin, 1979.

[59] L. TARTAR "Remarks on homogenization," Homogenization and effective moduli of materials and media (Minneapolis, Minn., 1984/1985), pp. 228-246, IMA Vol. Math. Appl., 1, Springer, New York-Berlin, 1986.

[60] L. Tartar "Estimations fines des coefficients homogénéisésés," Ennio De Giorgi colloquium (Paris, 1983), 168-187, Res. Notes in Math, 125, Pitman, Boston, MA-London, 1985.

[61] L. TaRTar "H-measures, a new approach for studying homogenisation, oscillations and concentration effects in partial differential equations," Proc. Roy. Soc. Edinburgh Sect. A 115, (1990), no. 3-4, 193-230.

[62] L. TARTAR "Remarks on the homogenization method in optimal design methods," Homogenization and applications to material sciences (Nice, 1995), pp. 393-412, GAKUTO Internat. Ser. Math. Sci. Appl. 9, Gakkōtosho, Tokyo, 1995.

[63] L. TARTAR "On mathematical tools for studying partial differential equations of continuum physics: H-measures and Young measures," Developments in partial differential equations and applications to mathematical physics, (Ferrara, 1991), pp. 201-217, Plenum Press, New York, 1992.

[64] L. TARTAR "H-measures and applications," Nonlinear partial differential equations and their applications. Collège de France Seminar, Vol. XI (Paris, 1989-1991), pp. 282-290, Pitman Res. Notes Math. Ser, 299, Longman Sci. Tech., Harlow, 1994.

[65] L. TARTaR "Beyond Young measures," Microstructure and phase transitions in solids (Udine, 1994). Meccanica 30 (1995), no. 5, 505-526.

[66] L. Tartar "Approximation of H-measures," Conference en honneur de Roland GLOWINSKI (Tours, May 1997); texte fini trop tard pour pouvoir être inclus dans les comptes rendus. Research Report 97-204, Department of Mathematical Sciences, CARNEGIE-MELLON University, Pittsburgh, PA 15213.

[67] L. C. Young Lectures on the calculus of variations and optimal control theory, W. B. Saunders Co., Philadelphia-London-Toronto, Ont. 1969.

Carnegie Mellon University, Pittsburgh, PA, 15213-3890, USA

http://www.math.cmu.edu/math/people/tartar.html 